\title{
Vitamin B12 deficiency, a cause of Bicytopenia complicating Pregnancy
}

\author{
${ }^{1}$ Nisha Bhatia, ${ }^{2}$ Krishna K Meka
}

\begin{abstract}
Introduction: Bicytopenia in pregnancy is a rare entity that poses as a diagnostic challenge. It may present as a combination of anemia, leukopenia or thrombocytopenia. Though bone marrow failure is the commonest cause, nutritional deficiencies need to be ruled out. We report a case of Bicytopenia in pregnancy due to Vitamin B12 deficiency.
\end{abstract}

Case report: A 22 year old primigravida with 29 weeks of gestation presented with severe anemia. On evaluation her haemoglobin was $5.1 \mathrm{gm} / \mathrm{dl}$ and blood picture showed a mixed population of normocytes, hypochromic red blood cells with macrocytes; normal neutrophil count with hypersegmented neutrophils and diminished platelets. Iron studies were normal and her serum Vitamin B12 levels were low. She was given blood transfusions to improve her hemoglobin. Post transfusion, she set into spontaneous labor and delivered a dead female baby of wt $1 \mathrm{~kg}$. Treatment with injectable cyanocobalamin followed by oral multivitamin led to a sustained improvement in the haematological parameters.

Discussion: This case demonstrates that B12 deficiency could be a rare cause of bicytopenia in pregnancy. Failure to diagnose and treat could lead to fatal maternal and fetal complications. Early diagnosis of B12 deficiency and supplementation is warranted.

Keywords: Bicytopenia, Complications in pregnancy, Severe anemia, Vitamin B12 deficiency.

How to cite this article: Bhatia N, Meka KK. Vitamin B12 Deficiency, a Cause of Bicytopenia complicating Pregnancy. World J Anemia 2018;2(1):39-40.

Source of support: Nil

Conflict of interest: None

\section{INTRODUCTION}

Bicytopenia is defined as a simultaneous deficiency of any two blood cell lines-red blood cells, white blood cells, or platelets-leading to anemia, leukopenia, or thrombocytopenia. It can adversely affect the health of the mother and fetus. Lower counts of multiple blood components

\footnotetext{
${ }^{1}$ Assistant Professor, ${ }^{2}$ Professor

1,2Department of Obstetrics and Gynecology, Apollo Institute of Medical Sciences \& Research, Hyderabad, Telangana, India

Corresponding Author: Krishna K Meka, Professor Department of Obstetrics and Gynecology, Apollo Institute of Medical Sciences \& Research, Hyderabad, Telangana, India Phone: +919849175200, e-mail: doc_krishnak@yahoo.co.in
}

in pregnancy are not only a diagnostic challenge but also difficult to treat, as it is life-threatening to both mother and the fetus. The common causes of bicytopenia in pregnancy are aplastic anemia, myelodysplastic syndrome, and megaloblastic anemia. ${ }^{1}$ Though bone marrow failure and malignancies are important causes, nutritional causes should be primarily ruled out. We report the rare case of a 22-year-old primigravida with bicytopenia due to vitamin B12 deficiency.

\section{CASE REPORT}

A 22-year-old primigravida with 29 weeks and 1 day of pregnancy was referred to the Department of Obstetrics and Gynecology, Apollo Institute of Medical Sciences \& Research, Hyderabad, Telangana, India, due to severe anemia for evaluation and management. She had conceived spontaneously after 1 year of marriage. She got booked in a private hospital at 4 months of gestation and had irregular antenatal visits. She got her antenatal investigations done for the first time at 28 weeks, which showed severe anemia-hemoglobin of $5.6 \mathrm{gm} \%$ - and hence was referred to our center for evaluation and management.

On presentation to our hospital, she was apparently asymptomatic, and a thorough history was taken. Her menstrual history was unremarkable. She had regular periods since menarche with normal flow. No history of melena or any other blood loss was seen. Though she consumed mixed diet, her diet was devoid of meat products, as they belonged to lower socioeconomic class. Her medical history was negative for any recent infection and drug intake. On clinical examination, the patient had pallor and raised jugular venous pressure. No edema, lymphadenopathy, or icterus were noted. The Cardiovascular system examination showed an ejection systolic murmur. Her lung fields were clear. Her vitals were stable. The obstetric examination showed uterus corresponding to 28 weeks of gestation with good fetal movements and heart sounds.

The patient's investigations were as follows: complete blood picture: hemoglobin $5.1 \mathrm{gm} / \mathrm{dL}$; hematocrit $18.2 \%$; red blood cell count 2.13 million $/ \mu \mathrm{L}$; total leukocyte count $6.26 \times 10^{3} / \mathrm{mm}$; platelet count $40,000 / \mathrm{cu} \mathrm{mm}$. Neutrophil count $56 \%$; lymphocytes $42 \%$. Peripheral blood smear showed mixed population of normocytic, normochromic, and hypochromic cells with macrocytes. Neutrophils 
showed normal count and hypersegmented neutrophils; platelets were diminished in count. Reticulocyte count 2.2. Iron studies: serum iron 109 (50-170 $\mu \mathrm{g} / \mathrm{dL}$ ); serum ferritin $169(12-150 \mathrm{ng} / \mathrm{mL})$; total iron-binding capacity 375 (45-85 $\mu \mathrm{mol} / \mathrm{L})$; serum transferrin 255 (200-350 mg/dL); serum B12 levels $150 \mathrm{pg} / \mathrm{mL}$; serum thyroid stimulating hormone was 1.7. Liver function tests and renal function tests were normal. Hematologist's opinion was taken.

The patient was given three units of packed cell transfusion to improve hemoglobin. The patient set into spontaneous labor at 29 weeks and delivered a dead female baby of weight $1 \mathrm{~kg}$. Since platelets were above $20,000 / \mathrm{cu} \mathrm{mm}$, the platelets were reserved and transfusion was deferred. The patient was treated with 1,000 $\mu \mathrm{g}$ of intramuscular cyanocobalamin daily for 1 week, followed by multivitamin supplements. Subsequently, platelet count and mean corpuscular volume normalized and there was sustained improvement in the hemoglobin level.

\section{DISCUSSION}

We have presented a case of a young primigravida with 29 weeks with severe anemia who had a blood picture showing macrocytes with low platelet count, due to vitamin B12 deficiency. She had a preterm delivery and delivered a dead baby. Bicytopenia-low hemoglobin and low platelet count-was found to be due to vitamin B12 deficiency. Vitamin B12 deficiency in this patient is most likely due to poor nutrition. Although she was a nonvegetarian, there was minimal intake of animal products in her diet.

Women with unexplained severe anemia with pancytopenia are diagnosed as B12 deficiency by hematological picture, normal iron studies, and serum B12 levels. Hypersegmentation of the neutrophils (more than 5 segments) can be seen. ${ }^{2,3}$ Serum B12 levels fall in pregnancy and thus are less reliable in assessing. ${ }^{4}$

The prevalence of vitamin B12 deficiency is high in the Indian population, with metabolic evidence of deficiency in $75 \%$ of young urban Indian men and women. ${ }^{5}$ Pregnant women in India have nutritional deficiencies, such as B12 deficiency, as their diet is deficient in nonvegetarian foods due to religious or socioeconomic reasons. ${ }^{6}$
The adverse effects of vitamin B12 deficiency depend upon its severity and its effect on fetus may range from abortion to low birth weight or intrauterine death of the fetus or congenital anomalies like neural tube defects. Lower vitamin B12 levels are associated with increased plasma homocysteine levels, which cause the above adverse effects. ${ }^{7}$

The treatment of vitamin B12 deficiency is the same as for nonpregnant patients. If there is no malabsorption, oral supplements of B12 are sufficient. Since absorption tests cannot be done in pregnancy, initially, B12 injections were given in our patient, followed by oral supplementation. ${ }^{8}$

\section{CONCLUSION}

Vitamin B12 deficiency rarely presents as a cause of bicytopenia and may pose a problem in diagnosis. If left untreated, it can cause fetal and maternal complications. Hence, looking beyond iron deficiency anemia is recommended in pregnant patients with severe anemia.

\section{REFERENCES}

1. Zhang C, Liang MY, Wang SM. Clinical analysis of bicytopenia and pancytopenia during pregnancy. Zhonghua Fu Chan Ke Za Zhi 2009 Jul;44(7):488-491.

2. Devalia V, Hamilton MS, Molloy AM; British Committee for Standards in Haematology. Guidelines for the diagnosis and treatment of cobalamin and folate disorders. Br J Haematol 2014 Aug;166(4):496-513.

3. Hvas AM, Nexo E. Diagnosis and treatment of vitamin B12 deficiency. Haematologica 2006 Nov;91(11):1506-1512.

4. Hudson B. 10 minute consultation: vitamin B12 deficiency. BMJ 2012 Jun;340:1245-1246.

5. Refsum H, Yajnik CS, Gadkari M, Schneede J, Vollset SE, Orning L, Guttormsen AB, Joglekar A, Sayyad MG, Ulvik A, et al. Hyperhomocysteinemia and elevated methylmalonic acid indicate a high prevalence of cobalamin deficiency in Asian Indians. Am J Clin Nutr 2001 Aug;74(2):233-241.

6. Yusufji D, Mathan VI, Baker SJ. Iron, folate and Vitamin B12 nutrition in pregnancy: a study of 1000 women from Southern India. Bull World Health Organ 1973;48(1):15-22.

7. Muthayya S, Kurpad AV, Duggan CP, Bosch RJ, Dwarkanath P, Mhaskar A, Mhaskar R, Thomas A, Vaz M, Bhat S, et al. Low maternal vitamin B12 status is associated with intrauterine growth retardation in urban South Indians. Eur J Clin Nutr 2006 Jun;60(6):791-801.

8. Idris N, Arsyad A. Vitamin B12 deficiency presenting as pancytopenia in pregnancy: a case report. Malays Fam Physician 2012 Aug;7(2-3):46-50. 